\title{
Random multiplicative functions: the Selberg-Delange class
}

\author{
Marco Aymone*
}

\begin{abstract}
Let $1 / 2 \leq \beta<1, p$ be a generic prime number and $f_{\beta}$ be a random multiplicative function supported on the squarefree integers such that $\left(f_{\beta}(p)\right)_{p}$ is an i.i.d. sequence of random variables with distribution $\mathbb{P}(f(p)=-1)=\beta=1-\mathbb{P}(f(p)=+1)$. Let $F_{\beta}$ be the Dirichlet series of $f_{\beta}$. We prove a formula involving measure-preserving transformations that relates the Riemann $\zeta$ function with the Dirichlet series of $F_{\beta}$, for certain values of $\beta$, and give an application. Further, we prove that the Riemann hypothesis is connected with the mean behavior of a certain weighted partial sum of $f_{\beta}$.
\end{abstract}

Keywords: random multiplicative functions; random Dirichlet series; Riemann hypothesis. MSC2020 subject classifications: Primary 60F15, Secondary 11N37.

Submitted to ECP on February 16, 2021, final version accepted on April 25, 2021.

\section{Introduction.}

We say that $f: \mathbb{N} \rightarrow \mathbb{C}$ is a multiplicative function if $f(n m)=f(n) f(m)$ for all nonnegative integers $n$ and $m$ with $\operatorname{gcd}(n, m)=1$, and that $f$ has support on the squarefree integers if for any prime $p$ and any integer power $k \geq 2, f\left(p^{k}\right)=0$. An important example of such functions is the Möbius function $\mu$, which is the multiplicative function supported on the squarefree integers such that the value at each prime $p$ is -1 .

Many important problems in Analytic Number Theory can be rephrased in terms of the mean behavior of the partial sums of multiplicative functions. For instance, the Riemann hypothesis - the statement that all the non-trivial zeros of the Riemann $\zeta$ function have real part equal to $1 / 2$ - is equivalent to the statement that the partial sums of the Möbius function have square root cancellation, that is, $\sum_{n \leq x} \mu(n)$ is $O_{\epsilon}\left(x^{1 / 2+\epsilon}\right)$, for all $\epsilon>0$. In this direction, the best unconditional result up to date is of the type $\sum_{n \leq x} \mu(n)=O\left(x \exp \left(-c(\log x)^{3 / 5}(\log \log x)^{1 / 5}\right)\right)$, for some constant $c>0$ (see Ivić [7], pp. 309-315). Any improvement of the type $\sum_{n \leq x} \mu(n)=O\left(x^{1-\epsilon}\right)$ for some $\epsilon>0$ would be a huge breakthrough in Analytic Number Theory, since it would imply that the Riemann $\zeta$ function has no zeros with real part greater than $1-\epsilon$.

This equivalence between the Riemann hypothesis with the mean behavior of the partial sums of the Möbius function led Wintner [12] to investigate the behavior of a random model $f$ for the Möbius function. This random model $f$ is defined as follows: $f$ is a random multiplicative function supported on the squarefree integers such that $(f(p))_{p \in \mathcal{P}}$ (here $\mathcal{P}$ stands for the set of primes) is an i.i.d. sequence of random variables whith distribution $\mathbb{P}(f(p)=-1)=\mathbb{P}(f(p)=+1)=1 / 2$. It is important to observe

\footnotetext{
*UFMG, Brazil. E-mail: aymone.marco@gmail.com
} 
that the sequence $(f(n))_{n \in \mathbb{N}}$ is highly dependent; for instance, since $30=2 \times 3 \times 5$, we have that $f(30)$ depends on the values $f(2), f(3)$ and $f(5)$. Wintner proved the square root cancellation for the partial sums of $f$, that is, $\sum_{n \leq x} f(n)=O\left(x^{1 / 2+\epsilon}\right)$ for all $\epsilon>0$, almost surely, and hence the assertion that the Riemann hypothesis is almost always true. This upper bound has been improved several times: [2], [4], [5] and [8]. The best upper bound up to date is due to Lau, Tenenbaum and Wu [8], which states that $\sum_{n \leq x} f(n)=O\left(\sqrt{x}(\log \log x)^{2+\epsilon}\right)$ for all $\epsilon>0$, almost surely, and the best $\Omega$ result is due to the recent result of Harper [6] which states that for any function $V(x)$ tending to infinity with $x$, there almost surely exist arbitrarily large values of $x$ for which $\left|\sum_{n \leq x} f(n)\right| \geq \sqrt{x} \frac{(\log \log x)^{1 / 4}}{V(x)}$.

Here we consider a slight different model for the Möbius function. We start with a parameter $1 / 2 \leq \beta \leq 1$ and consider a random multiplictive function $f_{\beta}$ supported on the squarefree integers and such that $\left(f_{\beta}(p)\right)_{p \in \mathcal{P}}$ is an i.i.d. sequence of random variables with $\mathbb{P}\left(f_{\beta}(p)=-1\right)=\beta=1-\mathbb{P}\left(f_{\beta}(p)=+1\right)$. For $\beta=1 / 2$, we recover the Wintner's model; for $\beta=1, f_{1}=\mu$; for $\beta<1, f_{\beta}(n)$ is equal to $\mu(n)$ with high probability as $\beta$ is taken to be close to 1 . In this paper we are interested in the following questions.

Question 1. What can be said about the partial sums $\sum_{n \leq x} f_{\beta}(n)$ for $1 / 2<\beta<1$ ? Do they have square root cancellation as in Wintner's model and as we expect for the Möbius function under the Riemann hypothesis?

Question 2. If the partial sums $\sum_{n \leq x} f_{\beta}(n)$ are $O\left(x^{1-\delta}\right)$ for some $\delta>0$, almost surely, then can we say something about the partial sums of the Möbius function?

Considering the first question, observe that $\mathbb{E} f_{\beta}(p)=1-2 \beta$, and thus, we might say that at primes, $f_{\beta}(p)$ is equal to $1-2 \beta$ on average. In the case $1 / 2<\beta<1$ the partial sums $\sum_{n \leq x} f_{\beta}(n)$ are well understood by the Selberg-Delange method, see the book of Tenenbaum [11] chapter II.5. Indeed, in the case that $1 / 2<\beta<1$, one can check that the Dirichlet series of $f_{\beta}$, say $F_{\beta}$, satisfies the required set of axioms for the Selberg-Delange method in [11] to apply. The most difficult to check is an upper bound in vertical strips for a random Dirichlet series with independent and mean zero summands $\sum_{n=1}^{\infty} \frac{X_{n}}{n^{s}}$, which has been done in [1]. Thus, the following holds almost surely

$$
\sum_{n \leq x} f_{\beta}(n)=\left(c_{f_{\beta}}+o(1)\right) \frac{x}{(\log x)^{2 \beta}}
$$

as $x \rightarrow \infty$, where $c_{f_{\beta}}$ is a random constant which is positive almost surely. In particular, this implies that $\sum_{n \leq x} f_{\beta}(n)$ is not $O\left(x^{1-\delta}\right)$, for any $\delta>0$, almost surely. This answers negatively to our question 1 .

Here we provide a more probabilistic proof that we do not have square root cancellation for $\sum_{n \leq x} f_{\beta}(n)$ for certain values of $\beta$, almost surely. Further, by considering the question 2, we show that the Riemann hypothesis is equivalent to the square root cancellation of certain weighted partial sums of $f_{\beta}$.

Before we state our results, let us introduce some notation. Given a probability space $(\Omega, \mathcal{F}, \mathbb{P})$, let $\omega$ be a generic element of $\Omega$, and $T: \Omega \rightarrow \Omega$ be a measurepreserving transformation, i.e., $\mathbb{P}\left(T^{-1}(A)\right)=\mathbb{P}(A)$, for all $A \in \mathcal{F}$. We look at the random multiplicative function $f_{\beta}$ defined over the probability space $(\Omega, \mathcal{F}, \mathbb{P})$ as a function $f_{\beta}: \mathbb{N} \times \Omega \rightarrow\{-1,0,1\}$, that is, $f_{\beta}(n)$ is a random variable such that $f_{\beta}(n, \omega) \in\{-1,0,1\}$. Moreover, the Dirichlet series of $f_{\beta}$, say $F_{\beta}(s):=\sum_{n=1}^{\infty} \frac{f_{\beta}(n)}{n^{s}}$, is a random analytic function over the half plane $\mathbb{H}_{1}:=\{s \in \mathbb{C}: \operatorname{Re}(s)>1\}$, that is $F_{\beta}: \mathbb{H}_{1} \times \Omega \rightarrow \mathbb{C}$ is such that $F_{\beta}(s, \omega)=\sum_{n=1}^{\infty} \frac{f_{\beta}(n, \omega)}{n^{s}}$ is analytic in the half plane $\mathbb{H}_{1}$, for all $\omega \in \Omega$.

Theorem 1.1. Let $n \geq 1$ be an integer, $\beta=1-\frac{1}{2^{n+1}}$, and $(\Omega, \mathcal{F}, \mathbb{P})$ be a certain probability space where it is defined $f_{\beta}$ for all values of $\beta \in[1 / 2,1]$. Let $F_{\beta}(s)=\sum_{n=1}^{\infty} \frac{f_{\beta}(n)}{n^{s}}$. Then there exists a measure-preserving transformation $T: \Omega \rightarrow \Omega$ such that $T^{2^{n}} \stackrel{n^{s}}{=}$ identity 
and such that the following formula holds for all $\operatorname{Re}(s)>1$ and all $\omega \in \Omega$ :

$$
\frac{1}{\zeta(s)^{2^{n}-1}}=\frac{1}{F_{1 / 2}(s, \omega)} \prod_{k=1}^{2^{n}} F_{\beta}\left(s, T^{k} \omega\right) .
$$

In particular, if $\beta=3 / 4$, we have

$$
\frac{1}{\zeta(s)}=\frac{F_{3 / 4}(s, \omega) F_{3 / 4}(s, T \omega)}{F_{1 / 2}(s, \omega)} .
$$

Corollary 1.2. For an integer $n \geq 1$ and $\beta=1-\frac{1}{2^{n+1}}$, we have that for any $\delta>0$, $\sum_{n \leq x} f_{\beta}(n)$ is not $O\left(x^{1-\delta}\right)$ almost surely.

The proof of corollary 1.2 utilizes the fact that the event in which $\sum_{n \leq x} f_{\beta}(n)=$ $O\left(x^{1-\delta}\right)$ is contained in the event in which the Dirichlet series $F_{\beta}(s)$ has analytic continuation to $\{\operatorname{Re}(s)>1-\delta\}$, from which, one can easily check that for $\beta>1 / 2, F_{\beta}(1)=0$ almost surely. In Wintner's proof [12] of the square root cancellation of $\sum_{n \leq x} f_{1 / 2}(n)$, it has been proved that $F_{1 / 2}(s)$ is almost surely a non-vanishing analytic function over the half plane $\{\operatorname{Re}(s)>1 / 2\}$. Thus, as $T$ preserves measure, the left side of (1.1) has a zero of multiplicity $2^{n}-1$ at $s=1$ while the right side of the same equation has a zero of multiplicity at least $2^{n}$ at the same point, which is a contradiction, and hence the event in which $F_{\beta}(s)$ has analytic continuation to $\{\operatorname{Re}(s)>1-\delta\}$ can not hold with probability 1. Moreover, by the Euler product formula for $\operatorname{Re}(s)>1$

$$
F_{\beta}(s)=\prod_{p \in \mathcal{P}}\left(1+\frac{f_{\beta}(p)}{p^{s}}\right),
$$

we see that the event in which $F_{\beta}$ has analytic continuation to $\{\operatorname{Re}(s)>1-\delta\}$ is a tail event, in the sense that it does not depend in any outcome on a finite number of the random variables $f_{\beta}\left(p_{1}\right), \ldots, f_{\beta}\left(p_{r}\right)$, where $p_{1}, \ldots, p_{r}$ are primes. The Kolmogorov zero-one law states that each tail event has probability either equal to 0 or to 1 . Thus, the event in which $F_{\beta}$ has analytic continuation to $\{\operatorname{Re}(s)>1-\delta\}$ has probability 0 , and hence the event in which $\sum_{n \leq x} f_{\beta}(n)=O\left(x^{1-\delta}\right)$ also has probability 0 .

Now we turn our attention to Question 2. As mentioned above, the event in which $\sum_{n \leq x} f_{\beta}(n)=O\left(x^{1-\delta}\right)$ for some $\delta>0$ has probability 0 . However, we can obtain an equivalence between the Riemann hypothesis and the mean behavior of certain weighted partial sums of $f_{\beta}$. Before we state our next result, let $\omega(n)$ be the number of distinct primes that divide $n$.

Theorem 1.3. The Riemann hypothesis is equivalent to the following statement:

$$
\sum_{n \leq x}(2 \beta-1)^{-\omega(n)} f_{\beta}(n)=O\left(x^{1 / 2+\epsilon}\right),
$$

for all $\epsilon>0$ and $x$ sufficiently large with respect to $\epsilon$, almost surely, for each $\frac{1}{2}+\frac{1}{2 \sqrt{2}}<$ $\beta<1$.

Here we describe the proof of Theorem 1.3. For all $\operatorname{Re}(s)>1$, we have the following formula:

$$
\sum_{n=1}^{\infty} \frac{(2 \beta-1)^{-\omega(n)} f_{\beta}(n)}{n^{s}}=\frac{1}{\zeta(s)} \exp \left(\sum_{p \in \mathcal{P}} \frac{(2 \beta-1)^{-1} f_{\beta}(p)+1}{p^{s}}+C_{\beta}(s)\right),
$$

where $C_{\beta}(\cdot)$ is a random function that is analytic almost surely in the half plane $\operatorname{Re}(s)>$ $1 / 2$ for each $\frac{1}{2}+\frac{1}{2 \sqrt{2}}<\beta<1$. If $\sum_{n \leq x}(2 \beta-1)^{-\omega(n)} f_{\beta}(n)=O\left(x^{1 / 2+\epsilon}\right)$, for all $\epsilon>0$, almost 
surely, then the function on the left-hand side of (1.3) is almost surely an analytic function in the half plane $\operatorname{Re}(s)>1 / 2$, and then we can conclude that $1 / \zeta(s)$ must be analytic in the same half plane, which implies the Riemann hypothesis. Now if the Riemann hypothesis is true, then the right-hand side of (1.3) is almost surely an analytic function in the half plane $\operatorname{Re}(s)>1 / 2$, which gives that the left-hand side of (1.3) has analytic continuation to $\operatorname{Re}(s)>1 / 2$, almost surely. It is noteworthy to notice that the existence of analytic continuation does not necessarily implies the convergence of a Dirichlet series. For instance, we have that $\eta(s):=\sum_{n=1}^{\infty} \frac{(-1)^{n+1}}{n^{s}}$ has analytic continuation to all of the complex plane and converges only in the half plane $\operatorname{Re}(s)>0$. However, in our case, we have the extra information that under the Riemann hypothesis, for all $\sigma \geq \sigma_{0}>1 / 2$ and all $t \in \mathbb{R}, 1 / \zeta(\sigma+i t)=O_{\sigma_{0}, \epsilon}\left(t^{\epsilon}\right)$, for all $\epsilon>0$, where the implicit constant in $O_{\sigma_{0}, \epsilon}$ depends only on $\sigma_{0}$ and $\epsilon$. Next, by Perron's formula, we can show that if a certain Dirichlet series has analytic continuation to a larger half plane, and in this half plane satisfies the $O\left(t^{\epsilon}\right)$-bound above, then this series converges in this larger half plane. Thus, all we need to do is to bound the random Dirichlet series over primes $P(s):=\sum_{p \in \mathcal{P}} \frac{(2 \beta-1)^{-1} f_{\beta}(p)+1}{p^{s}}$ in vertical strips. More precisley, we need to verify a bound roughly of the type $P(\sigma+i t)=o(\log t)$, for each fixed $\sigma>1 / 2$, almost surely. This has been done by Carlson for Rademacher summands in [3], where he showed the almost sure bound $O(\sqrt{\log t})$, and then improved to $O\left((\log t)^{1-\sigma} \log \log t\right)$ and to general random variables satisfying some moment conditions by Sidoravicius and the author in [1].

\section{Preliminaries}

\subsection{Notations}

Here we let $p$ denote a generic prime number and $\mathcal{P}$ the set of primes. We use $f(x) \ll g(x)$ and $f(x)=O(g(x))$ whenever there exists a constant $c>0$ such that $|f(x)| \leq c|g(x)|$, for all $x$ in a certain set $X$ - This set $X$ could be all the interval $[1, \infty)$ or $(a-\delta, a+\delta), a \in \mathbb{R}, \delta>0$. We say that $f(x)=o(g(x))$ if $\lim _{x \rightarrow \infty} \frac{f(x)}{g(x)}=0$. The notation $d \mid n$ means that $d$ divides $n$. Here $*$ stands for the Dirichlet convolution $(f * g)(n):=\sum_{d \mid n} f(d) g(n / d)$. We denote $\omega(n)=\sum_{p \mid n} 1$, that is, the number of distinct primes that divide $n$. In some contexts, the letter $\omega$ will also denote a random element of a certain set of realizations $\Omega$.

\section{Proof of the results}

\subsection{Construction of the probability space}

We let $\mathcal{P}$ be the set of primes, $\Omega=[0,1]^{\mathcal{P}}=\left\{\omega=\left(\omega_{p}\right)_{p \in \mathcal{P}}: \omega_{p} \in[0,1]\right.$ for all $\left.p\right\}, \mathcal{F}$ the Borel sigma algebra of $\Omega$ and $\mathbb{P}$ be the Lebesgue measure in $\mathcal{F}$. We set $f_{\beta}(p)$ as

$$
f_{\beta}\left(p, \omega_{p}\right)=-\mathbb{1}_{[0, \beta]}\left(\omega_{p}\right)+\mathbb{1}_{(\beta, 1]}\left(\omega_{p}\right) .
$$

It follows that $\left(f_{\beta}(p)\right)_{p \in \mathcal{P}}$ are i.i.d. with distribution $\mathbb{P}\left(f_{\beta}(p)=-1\right)=\beta=1-\mathbb{P}\left(f_{\beta}(p)=\right.$ +1 ). Also, we say that $f_{\beta}$ are uniformly coupled for different values of $\beta$, since $f_{\beta}(p)$ can be written as $f_{\beta}(p)=\lambda\left(U_{p}, \beta\right)$, where $\lambda$ is a function $\lambda:[0,1]^{2} \rightarrow \mathbb{R}$ and $U_{p}$ is a random variable with uniform distribution on the interval $[0,1]$.

\subsection{Construction of the measure-preserving transformation}

Now if $\beta=1-\frac{1}{2^{n+1}}$ with $n \geq 1$ an integer, we partionate the interval $[1 / 2,1]$ into $2^{n}$ subintervals $I_{k}=\left(a_{k-1}, a_{k}\right)$ of length $\frac{1}{2^{n+1}}$ and with endpoints $a_{k}=\frac{1}{2}+\frac{k}{2^{n+1}}$. It follows that $a_{0}=1 / 2, a_{2^{n}-1}=\beta$ and $a_{2^{n}}=1$.

Let $T_{p}:[0,1] \rightarrow[0,1]$ be the following interval exchange transformation: for $\omega_{p} \in$ $[0,1 / 2], T_{p}\left(\omega_{p}\right)=\omega_{p}$; in each interval $I_{k}$ as above the restriction $\left.T_{p}\right|_{I_{k}}$ is a translation; 
$T_{p}\left(I_{1}\right)=I_{2^{n}}$ and for $k \geq 2, T_{p}\left(I_{k}\right)=I_{k-1}$. It follows that the $k$ th iterate $T_{p}^{k}\left(I_{k}\right)=I_{2^{n}}$ and $T_{p}^{2^{n}}$ is the identity. Also, for each prime $p, T_{p}$ and its iterates preserve the Lebesgue measure and hence, $T: \Omega \rightarrow \Omega$ defined by $T \omega:=\left(T_{p}\left(\omega_{p}\right)\right)_{p \in \mathcal{P}}$ preserves $\mathbb{P}$, and so do its iterates.

\subsection{Proof of Theorem 1.1}

Proof. We let $F_{\beta}$ be the Dirichlet series of $f_{\beta}$ and $I_{k}=\left(a_{k-1}, a_{k}\right]$ be as above. Notice that $a_{0}=1 / 2$ and $a_{2^{n}}=1$, and hence $F_{a_{0}}=F_{1 / 2}$ and $F_{a_{2^{n}}}=F_{1}=\frac{1}{\zeta}$. Observe that

$$
F_{1 / 2} \zeta=\frac{F_{a_{0}}}{F_{a_{2^{n}}}}=\frac{F_{a_{0}}}{F_{a_{1}}} \cdot \frac{F_{a_{1}}}{F_{a_{2}}} \cdot \ldots \cdot \frac{F_{a_{2^{n}-1}}}{F_{a_{2^{n}}}} .
$$

Now, by the Euler product formula (1.2), we have that for all $\operatorname{Re}(s)>1$

$$
\frac{F_{a_{k}}}{F_{a_{k+1}}}(s, \omega)=\prod_{p \in \mathcal{P}} \frac{1+\frac{f_{a_{k}}\left(p, \omega_{p}\right)}{p^{s}}}{1+\frac{f_{a_{k+1}}\left(p, \omega_{p}\right)}{p^{s}}}=\prod_{p \in \mathcal{P}} \frac{p^{s}+\mathbb{1}_{I_{k+1}}\left(\omega_{p}\right)}{p^{s}-\mathbb{1}_{I_{k+1}}\left(\omega_{p}\right)} .
$$

Thus, as all intervals $I_{k}$ have same length, we see that each $\frac{F_{a_{k}}}{F_{a_{k+1}}}$ is equal in probability distribution to the last $\frac{F_{a_{2^{n}}-1}}{F_{a_{2^{n}}}}$. Moreover, if $T$ is as above, since $\mathbb{1}_{I_{k}}\left(\omega_{p}\right)=\mathbb{1}_{I_{2^{n}}} \circ T_{p}^{k}\left(\omega_{p}\right)$, we have that

$$
\frac{F_{a_{k}}}{F_{a_{k+1}}}(s, \omega)=\frac{F_{a_{2^{n}-1}}}{F_{a_{2^{n}}}}\left(s, T^{k+1} \omega\right)=F_{\beta}\left(s, T^{k+1} \omega\right) \zeta(s) .
$$

Thus

$$
F_{1 / 2}(s, \omega) \zeta(s)=\zeta(s)^{2^{n}} \prod_{k=1}^{2^{n}} F_{\beta}\left(s, T^{k} \omega\right),
$$

which concludes the proof.

\subsection{Proof of Corollary 1.2}

Proof. A standard result about Dirichlet series is that the Dirichlet series of an arithmetic function $f$, say $F(s)$, is the Mellin transform of the partial sums of $f$. Indeed, we have that for $s$ in the half plane of convergence of $F(s)$,

$$
F(s)=s \int_{1}^{\infty} \frac{\sum_{n \leq x} f(n)}{x^{s+1}} d x .
$$

Thus, we can conclude that the event in which the partial sums $\sum_{n \leq x} f(n)$ are $O\left(x^{\alpha}\right)$ is contained in the event in which the Dirichlet series $F(s):=\sum_{n=1}^{\infty} \frac{f(n)}{n^{s}}$ is analytic in the half plane $\{\operatorname{Re}(s)>\alpha\}$. Thus, under the assumption that $\sum_{n \leq x} f_{\beta}(n)=O\left(x^{1-\delta}\right)$ almost surely, we have that $F_{\beta}(s)=\sum_{n=1}^{\infty} \frac{f_{\beta}(n)}{n^{s}}$ has analytic continuation to the half plane $\{\operatorname{Re}(s)>1-\delta\}$ almost surely. Moreover, we can check that $F_{\beta}(1)=0$ almost surely. Indeed, by taking the logarithm of the Euler product formula (1.2) and then using Taylor expansion for each logarithm, we see that

$$
F_{\beta}(s)=\exp \left(\sum_{p \in \mathcal{P}} \frac{f_{\beta}(p)}{p^{s}}+A_{\beta}(s)\right),
$$

where $A_{\beta}(s)=O_{\sigma_{0}}(1)$ for all $\operatorname{Re}(s) \geq \sigma_{0}>1 / 2$. Since $\mathbb{E} f_{\beta}(p)=1-2 \beta<0$ for all primes $p$, we have by the Kolmogorov two series theorem that $\lim _{s \rightarrow 1^{+}} \sum_{p \in \mathcal{P}} \frac{f_{\beta}(p)}{p^{s}}=-\infty$ almost surely, and hence, $\lim _{s \rightarrow 1^{+}} F_{\beta}(s)=0$ almost surely. 
If $T$ is the measure-preserving transformation as in Theorem 1.1, then the same is almost surely true for $F_{\beta}\left(s, T^{k} \omega\right)$. Further, in the Wintner's proof [12] of the square root cancellation of $\sum_{n \leq x} f_{1 / 2}(n)$, it has been proved that $F_{1 / 2}(s)$ is almost surely a non-vanishing analytic function over the half plane $\{\operatorname{Re}(s)>1 / 2\}$. Indeed, this can be proved by the formula (3.1).

A well known fact is that the Riemann $\zeta$ function has a simple pole at $s=1$, and hence, $\frac{1}{\zeta(s)}$ has a simple zero at the same point. Moreover, we recall that if an analytic function $G$ has a zero at $s=s_{0}$, then there exists a non-vanishing analytic function $H$ at $s=s_{0}$ and a non-negative integer $m$, called the multiplicity of the zero $s_{0}$, such that $G(s)=\left(s-s_{0}\right)^{m} H(s)$. Thus the left-hand side of

$$
\frac{1}{\zeta(s)^{2^{n}-1}}=\frac{1}{F_{1 / 2}(s, \omega)} \prod_{k=1}^{2^{n}} F_{\beta}\left(s, T^{k} \omega\right)
$$

has a zero of multiplicity $2^{n}-1$ at $s=1$, while the right-hand side of the same equation has a zero of multiplicity at least $2^{n}$ at the same point, almost surely, which is a contradiction. Thus we see that the probability of the event in which $F_{\beta}(s)$ has analytic continuation to $\operatorname{Re}(s)>1-\delta$ is strictly less than one. Now we can check by the Euler product formula (1.2) that the event in which $F_{\beta}$ has analytic continuation to $\operatorname{Re}(s)>1-\delta$ is a tail event for $\delta<1$, i.e., whether $F_{\beta}$ has analytic continuation to $\{\operatorname{Re}(s)>1-\delta\}$ does not depend in any outcome of a finite number of random variables $\left\{f_{\beta}(p): p \leq y\right\}$. Indeed, we can write

$$
F_{\beta}(s)=\prod_{p \leq y}\left(1+\frac{f_{\beta}(p)}{p^{s}}\right) \prod_{p>y}\left(1+\frac{f_{\beta}(p)}{p^{s}}\right)
$$

and since $\prod_{p \leq y}\left(1+\frac{f_{\beta}(p)}{p^{s}}\right)$ is a non-vanishing analytic function in $\operatorname{Re}(s)>0$, we obtain that $F_{\beta}(s)$ has analytic continuation to $\operatorname{Re}(s)>1-\delta(\delta<1)$ if and only if $X_{y}(s):=\prod_{p>y}\left(1+\frac{f_{\beta}(p)}{p^{s}}\right)$ has analytic continuation to the same half plane. Since $X_{y}(s)$ is independent of $\left\{f_{\beta}(p): p \leq y, p \in \mathcal{P}\right\}$ and the random variables $\left(f_{\beta}(p)\right)_{p \in \mathcal{P}}$ are independent, we conclude that the event in which $F_{\beta}$ has analytic continuation to $\{\operatorname{Re}(s)>1-\delta\}$ is a tail event.

Thus by the Kolmogorov zero-one law, we have that the probability that $F_{\beta}$ has analytic continuation to $\{\operatorname{Re}(s)>1-\delta\}$ is zero, and hence the probability of $\sum_{n \leq x} f_{\beta}(n)=O\left(x^{1-\delta}\right)$ is also zero.

\subsection{Proof of Theorem 1.3}

Proof. We begin by observing that the function $g_{\beta}(n):=(2 \beta-1)^{-\omega(n)} f_{\beta}(n)$ is multiplicative and supported on the squarefree integers. Moreover, at each prime $p, g_{\beta}(p)=\frac{f_{\beta}(p)}{2 \beta-1}$, and hence $\mathbb{E} g_{\beta}(p)=-1$. If $\beta>\frac{1}{2}+\frac{1}{2 \sqrt{2}}$, we have that

$$
A_{\beta}(s):=\sum_{p \in \mathcal{P}} \sum_{m=2}^{\infty} \frac{(-1)^{m+1}}{m} \frac{g_{\beta}(p)^{m}}{p^{m s}}
$$

converges absolutely for all $\operatorname{Re}(s)>1 / 2$ and hence defines a random analytic function in this half plane. Moreover, $A_{\beta}(s)=O_{\sigma_{0}}(1)$ uniformly for all $\operatorname{Re}(s) \geq \sigma_{0}>1 / 2$. Thus, by the Euler product formula (1.2) for $g_{\beta}$, we have that the Dirichlet series $G_{\beta}(s):=\sum_{n=1}^{\infty} \frac{g_{\beta}(n)}{n^{s}}$ can be represented in the half plane $\operatorname{Re}(s)>1$ as

$$
G_{\beta}(s)=\exp \left(\sum_{p \in \mathcal{P}} \frac{g_{\beta}(p)}{p^{s}}+A_{\beta}(s)\right) .
$$


Moreover, by the same argument, there exists an analytic function $B(s)$ with the same properties of $A_{\beta}(s)$ such that

$$
\zeta(s)=\exp \left(\sum_{p \in \mathcal{P}} \frac{1}{p^{s}}+B(s)\right)
$$

Now observe that

$$
H_{\beta}(s):=G_{\beta}(s) \zeta(s)=\exp \left(\sum_{p \in \mathcal{P}} \frac{g_{\beta}(p)+1}{p^{s}}+A_{\beta}(s)+B(s)\right) .
$$

Now, by the Kolmogorov two series theorem, $\sum_{p \in \mathcal{P}} \frac{g_{\beta}(p)+1}{p^{s}}$ converges almost surely for all $\operatorname{Re}(s)>1 / 2$ and hence it defines, almost surely, a random analytic function in this half plane. Moreover, by Theorem 3.1 of [1], for fixed $1 / 2<\sigma \leq 1$, we have that for all large $t>0, \sum_{p \in \mathcal{P}} \frac{g_{\beta}(p)+1}{p^{\sigma+i t}} \ll(\log t)^{1-\sigma} \log \log t$, almost surely. Thus, for each fixed $1 / 2<\sigma$, we have

$$
H_{\beta}(\sigma+i t), 1 / H_{\beta}(\sigma+i t) \ll t^{\epsilon},
$$

for all $\epsilon>0$ and $t$ sufficiently large with respect to $\epsilon$, almost surely. A well known consequence of the Riemann hypothesis, is that $1 / \zeta(s)$ has analytic continuation to $\operatorname{Re}(s)>1 / 2$ and for each fixed $\sigma>1 / 2,1 / \zeta(\sigma+i t) \ll t^{\epsilon}$, for all $\epsilon>0$ and $t$ sufficiently large with respect to $\epsilon$. Thus, if we assume the Riemann hypothesis, we obtain that $G_{\beta}(s)$ has analytic continuation to $\operatorname{Re}(s)>1 / 2$ given by $G_{\beta}(s)=H_{\beta}(s) / \zeta(s)$ and for each fixed $\sigma>1 / 2, G_{\beta}(\sigma+i t) \ll t^{\epsilon}$ for all $\epsilon>0$ and $t$ sufficiently large with respect to $\epsilon$, almost surely. The last bound holds, almost surely, uniformly in the half plane $\sigma \geq \sigma_{0}>1 / 2$; see for instance [11], Chapter II.1, Theorem 1.20 and the Remark after.

Now we recall the Perron's formula (see [9], Theorem 5.2 and Corollary 5.3): for $T>0$,

$$
\sum_{n \leq x} g_{\beta}(n)=\int_{2-i T}^{2+i T} G_{\beta}(s) \frac{x^{s}}{s} d s+O\left(x^{1 / 4}+\frac{x^{2}}{T}\right) .
$$

Let $1 / 2<\sigma<1$ and let $\mathcal{R}$ be the rectangle with vertices $2-i T, 2+i T, \sigma+i T$ and $\sigma-i T$. By the Cauchy integral formula, almost surely

$$
\int_{2-i T}^{2+i T} G_{\beta}(s) \frac{x^{s}}{s} d s=-\int_{2+i T}^{\sigma+i T} G_{\beta}(s) \frac{x^{s}}{s} d s-\int_{\sigma+i T}^{\sigma-i T} G_{\beta}(s) \frac{x^{s}}{s} d s-\int_{\sigma-i T}^{2-i T} G_{\beta}(s) \frac{x^{s}}{s} d s .
$$

Now

$$
\int_{2+i T}^{\sigma+i T} G_{\beta}(s) \frac{x^{s}}{s} d s \ll \frac{1}{T^{1-\epsilon}} \int_{\sigma}^{2} x^{\sigma} d x \ll \frac{x^{2}}{T^{1-\epsilon}}
$$

and similarly

$$
\int_{\sigma-i T}^{2-i T} G_{\beta}(s) \frac{x^{s}}{s} d s \ll \frac{x^{2}}{T^{1-\epsilon}}
$$

Further

$$
\int_{\sigma+i T}^{\sigma-i T} G_{\beta}(s) \frac{x^{s}}{s} d s \ll T^{\epsilon} x^{\sigma} \int_{-T}^{T} \frac{d t}{|\sigma+i t|} \ll T^{2 \epsilon} x^{\sigma} .
$$

By combining these estimates, we obtain that

$$
\sum_{n \leq x} g_{\beta}(n) \ll T^{2 \epsilon} x^{\sigma}+\frac{x^{2}}{T^{1-\epsilon}},
$$


almost surely. By selecting $T=x^{3}$ and $\epsilon>0$ small enough, we obtain that the right-hand side of the above (3.2) is $\ll x^{\sigma+6 \epsilon}$, if $x$ is sufficiently large with respect to $\epsilon$. By making $\sigma \rightarrow 1 / 2^{+}$, we get the desired almost sure bound.

To prove the other implication, if $\sum_{n \leq x} g_{\beta}(n) \ll x^{1 / 2+\epsilon}$ for all $\epsilon>0$ and $x$ sufficiently large with respect to $\epsilon$, almost surely, then $G_{\beta}(s)$ is almost surely analytic in $\operatorname{Re}(s)>1 / 2$ and thus $G_{\beta}(s) / H_{\beta}(s)$ also is almost surely analytic in $\operatorname{Re}(s)>1 / 2$. Since $1 / \zeta(s)=$ $G_{\beta}(s) / H_{\beta}(s)$, we have that $1 / \zeta(s)$ has analytic continuation to $\operatorname{Re}(s)>1 / 2$. This last assertion is equivalent to the Riemann hypothesis.

\section{References}

[1] M. Aymone and V. Sidoravicius, Partial sums of biased random multiplicative functions, J. Number Theory, 172 (2017), pp. 343-382. MR-3573158

[2] J. Basquin, Sommes friables de fonctions multiplicatives aléatoires, Acta Arith., 152 (2012), pp. 243-266. MR-2885786

[3] F. Carlson, Contributions à la théorie des séries de Dirichlet. III, Ark. Mat. Astr. Fys., 23A, 19 (1933). MR-0051334

[4] P. Erdős, Some unsolved problems, Magyar Tud. Akad. Mat. Kutató Int. Közl., 6 (1961), pp. 221-254. MR-0177846

[5] G. Halász, On random multiplicative functions, in Hubert Delange colloquium (Orsay, 1982), vol. 83 of Publ. Math. Orsay, Univ. Paris XI, Orsay, 1983, pp. 74-96. MR-0728404

[6] A. J. Harper, Almost sure large fluctuations of random multiplicative functions, arXiv:2012.15809, 2021

[7] A. Ivić, The Riemann zeta-function , John Wiley \& Sons, Inc., New York, 1985. MR-0792089

[8] Y.-K. Lau, G. Tenenbaum, and J. Wu, On mean values of random multiplicative functions, Proc. Amer. Math. Soc., 141 (2013), pp. 409-420. MR-2996946

[9] H. L. Montgomery and R. C. Vaughan, Multiplicative number theory. I. Classical theory, vol. 97 of Cambridge Studies in Advanced Mathematics, Cambridge University Press, Cambridge, 2007. MR-2378655

[10] A. N. Shiryaev, Probability, vol. 95 of Graduate Texts in Mathematics, Springer-Verlag, New York, second ed., 1996. Translated from the first (1980) Russian edition by R. P. Boas. MR-1368405

[11] G. Tenenbaum, Introduction to analytic and probabilistic number theory, vol. 46 of Cambridge Studies in Advanced Mathematics, Cambridge University Press, Cambridge, 1995. Translated from the second French edition (1995) by C. B. Thomas. MR-1342300

[12] A. Wintner, Random factorizations and Riemann's hypothesis, Duke Math. J., 11 (1944), pp. 267-275. MR-0010160

Acknowledgments. I would like to thank the anonymous referee for a careful reading of the paper and for useful suggestions and corrections. The content of this paper is part of the author's Phd Thesis. I would like to thank my Phd supervisor, Prof. Vladas Sidoravicius, for his encouragement, great intuition, ideas and enthusiasm that played a fundamental role in my academic trajectory. 